\title{
APORTACIONES Y LAGUNAS DE LA POLÍTICA MARÍTIMA INTEGRADA PARA ATAJAR LA VULNERABILIDAD DE LOS MARES Y OCÉANOS FRENTE AL AVANCE DEL CRECIMIENTO AZUL
}

\author{
INPUTS AND FAULTS OF THE INTEGRATED MARITIME POLICY TO \\ REDUCE THE VULNERABILITY OF THE SEAS AND OCEANS AGAINST THE \\ ADVANCE OF BLUE GROWTH
}

ANA María Aldaz CASANOVA

Profesora Asociada de Derecho Internacional Público

Universidad de Murcia

ana.aldaz@um.es

Fecha de recepción: 12/02/2021 - Fecha de aceptación: 03/04/2021

RESUMEN: El presente trabajo se proyecta sobre la política marítima integrada diseñada por la Unión Europea, con miras a cotejarla desde los criterios de la sostenibilidad que la propia Unión se ha impuesto y contrastar su eficacia y utilidad para la consecución de unos objetivos de calidad medioambiental de los mares y océanos que a día de hoy se formulan con carácter imperioso y urgente.

Palabras clave: Directiva Marco sobre Estrategia Marina, protección medioambiental, mares y océanos, desarrollo sostenible, Crecimiento Azul, política marítima integrada, Pacto Verde Europeo.

ABSTRACT: This paper addresses the integrated maritime policy designed by the European Union to compare it against the criteria of sustainability that the Union has self-imposed. The aim is to test its effectiveness and usefulness in achieving the imperative environmental quality objectives of the seas and oceans

Keywords: Marine Strategy Framework Directive, environmental protection, seas and oceans, sustainable development, Blue Growth, integrated maritime policy, European Green Deal.

SUMARIO: I. EVOLUCiÓN DEL DERECHO EUROPEO EN CLAVE ECOLÓGICA. DEL DESARROLlO ECONÓMICO AL DESARROLLO SOSTENIBLE. LA PROMESA DEL PACTO VERDE EUROPEO. II. INICIATIVAS DE CRECIMIENTO AZUL Y POLÍTICA MARÍTIMA INTEGRADA EN LA UE. III. BALANCE DE LA POLÍTICA MARÍTIMA INTEGRADA 2002-2020. EL IMPERATIVO DE UN CAMBIO DE PARADIGMA REAL. IV. REFERENCIAS BIBLIOGRÁFICAS. 


\section{EVOLUCIÓN DEL DERECHO EUROPEO EN CLAVE ECOLÓGICA. DEL DESARROLLO ECONÓMICO AL DESARROLLO SOSTENIBLE. LA PROMESA DEL PACTO VERDE EUROPEO ${ }^{1}$}

Si nos retrotraemos al momento de creación de la Unión Europea, a nadie se le escapa que la intencionalidad perseguida en su día por los Estados fundadores se orientó inicialmente hacia una vertiente esencialmente económica, en un contexto de post-guerra en el que la miseria y la crisis económica estaban causando estragos en el continente. Esa finalidad primigenia esencialmente material que respondía a las necesidades básicas propias del contexto histórico, se tradujo en los primeros tratados, que no solo no recogían mención alguna a la protección del medio ambiente, incluso presentaban una lacerante carencia respecto de la mención de los derechos humanos en general ${ }^{2}$.

Sin embargo, dando respuesta a toda una serie de declaraciones internacionales y regionales proclamadas al auspicio de nuevas demandas sociales que reclamaban una comunidad europea en la que las consideraciones medioambientales figuraran como pilar esencial en la toma de decisiones ${ }^{3}$, a finales del siglo XX se facilitó la aprobación de algunos actos jurídicos con contenido ambiental ${ }^{4}$. En esta línea, la Unión Europea terminó acogiendo un nuevo paradigma del orden socioeconómico internacional, que se recoge en varios artículos de su derecho Constitutivo y se formula como objetivo imperioso a alcanzar en el ámbito de la Unión ${ }^{5}$. Se pergeña así un modelo de desarrollo orientado a "vivir bien respetando los límites de nuestro planeta".

En este contexto, el Tratado de la Unión Europea matiza y complementa las primigenias aspiraciones eminentemente económicas y contempla en su artículo 3 un modelo de desarrollo

\footnotetext{
${ }^{1}$ Todas las citas de sitios de internet que se realizan a lo largo del presente trabajo han tenido como fecha de última consulta el 30 de marzo de 2021.

${ }^{2}$ LIÑÁN NOGUERAS, D. J. y MARTÍN RODRÍGUEZ, P. J., "Reflexiones sobre los derechos fundamentales de la Unión europea a la luz del Tratado de Lisboa", en BADIA MARTÍ, A .M., PIGRAU SOLÉ, A. y OLESTI RAYO, A. (Coord.), Derecho Internacional y comunitario ante los retos de nuestro tiempo. Homenaje a la profesora Victoria Abellán Honrubia, Marcial Pons, Madrid, 2009, p. 1059. Véase también, MANGAS MARTÍN, A., "El compromiso con los derechos fundamentales", en MANGAS MARTÍN, A., GONZÁLEZ ALONSO, L. N., LÓPEZ ESCUDERO, M., MARTÍN Y PEREZ DE NANCLARES, J. y SOBRINO HEREDIA, J. M., Carta de los Derechos Fundamentales de la Unión Europea. Comentario artículo por artículo, Fundación BBVA, Bilbao 2008., pp. 33 y ss.; y VITORINO, A., "La Cour de justice et les droits fondamentaux depuis la proclamation de la Charte", en COLNERIC, N., PUISSOCHET, J. P., RUIZ-JARABO Y COLOMER, D. y EDWARDS, D. V., Une communauté de droit: Festschrift für Gil Carlos Rodríguez Iglesias, BWV Berliner Wissenshafts, Berlin, 2003, pp. 111-126.
}

${ }^{3}$ Sobre la historia del nacimiento de la política ambiental comunitaria, véase FERNÁNDEZ DE GATTA SÁNCHEZ, D., "La política ambiental comunitaria: especial referencia a los programa ambientales", Revista de Instituciones Europeas, núm. 3, 1985, pp. 723-753; FERNÁNDEZ DE GATTA SÁNCHEZ, D., "La política ambiental comunitaria: su evolución y su futuro", Revista de Administración Pública, núm. 111, 1986, pp. 425-440; LÓPEZ RAMÓN, F., "La política de medio ambiente de la Comunidad Europea y su incidencia en el derecho español", en GARCÍA DE ENTERRÍA, E., GONZÁLEZ CAMPOS, J. D. y MUÑ̃Z MACHADO, S., (Dir.), Tratado de Derecho Comunitario Europeo, núm. III, Civitas, Madrid, 1986 y MARTÍN MATEO, R., "El ambiente en la CEE", Noticias de la Unión Europea, núm. 14, 1986, pp. 47-48.

${ }^{4}$ DÍEZ DE VELASCO VALLEJO, M., "Aspectos jurídicos actuales de la protección del medioambiente en la Comunidad Europea y, en especial, la contribución de su Tribunal de Justicia”, en RODRÍGUEZ IGLESIAS, G. y LIÑÁN NOGUERAS, D. (coord.), El derecho comunitario europeo y su aplicación judicial, Civitas, Madrid, 1993, pp. 1003-1028. En el mismo sentido, ESPÓSITO MASSICCI, C. y FERNÁNDEZ EGEA, R. M., "La protección del medio ambiente a través de los derechos exclusivos y el derecho de la competencia. A propósito de la sentencia del Tribunal de Justicia de las Comunidades Europeas de 23 de mayo de 2000", Gaceta jurídica de la Unión Europea y la competencia, núm. 211, 2001, p. 55.

${ }^{5}$ ESPÓSITO MASSICCI, C. y FERNÁNDEZ EGEA, R. M., “La protección del medio ambiente...”, cit., p. 57.

6 VII PMA. "Programa General de Acción de la Unión en materia de Medio Ambiente hasta 2020". Disponible en https://ec.europa.eu/environment/efe/news/here-2020-eus-new-environment-action-programme-2014-04-29 es 
que se habrá de edificar sobre tres pilares de dispar naturaleza, por un lado las originarias ambiciones propiamente económicas, como el crecimiento económico equilibrado y la estabilidad de los precios, por otro condicionantes sociales, como la economía social tendente al pleno empleo y el progreso social, y por último las demandas medioambientales que reclaman un elevado nivel de protección del medio ambiente. Se consagra así el desarrollo sostenible como una servidumbre que se perfila como un objetivo esencial ${ }^{7}$ y principio marco que rige en todas sus actuaciones ${ }^{8}$.

Sin embargo esta ecologización del Derecho de la Unión hacia la sostenibilidad no supone cambio de rumbo con respecto al camino ya trazado desde sus orígenes por el modelo de capitalismo dogmático ${ }^{9}$ y su diseño e implementación no quedan alejados de su sustento, que lleva a la incentivación de la producción y del consumo. Como consecuencia, este giro de las políticas europeas hacia la sostenibilidad no ha podido evitar, ni siquiera atajar, la grave crisis ecológica y sus devastadoras consecuencias en la biodiversidad terrestre y marina ${ }^{10}$.

En este contexto emerge el Pacto Verde Europeo $^{11}$ en diciembre de 2019 como una "nueva estrategia" que ambiciona transformar la Unión Europea en una sociedad justa y próspera en la que el crecimiento económico estará disociado del uso de los recursos, para lo que destaca la importancia de la participación activa del público y su confianza en esta transición y una política de consumidores que contribuya a su empoderamiento para que "tomen sus decisiones con conocimiento de causa y participen activamente en la transición ecológica". Se aboga en definitiva por un nuevo pacto que asocie ciudadanos, autoridades nacionales, sociedad civil, sectores económicos, instituciones de la Unión y órganos consultivos. Instituyendo a esta iniciativa como una "nueva senda" de crecimiento sostenible e integrador. Procede preguntarse en este punto si esta inquietud "transformadora" que se anuncia en el Pacto verde ha marcado ya una correlativa impronta en la revisión de las políticas y estrategias lideradas hasta la fecha por la Unión europea.

\section{INICIATIVAS DE CRECIMIENTO AZUL Y POLÍTICA MARÍTIMA INTEGRADA EN LA UE}

Los océanos y mares han constituido tradicionalmente piezas clave y motores esenciales de la economía mundial ${ }^{12}$, ofreciendo una promesa de prosperidad económica ante la

${ }^{7}$ El Abogado General Sr. Dámaso Ruiz-Jarabo Colomer en sus conclusiones presentadas el 26 de mayo de 2005 en el caso C-176/03, Sentencia de 13 de septiembre de 2005, Comisión/Consejo, C-176/03, EU:C:2005:542, califica la protección medioambiental de "designio esencial del sistema comunitario".

${ }^{8}$ En relación a la interrelación opuesta entre desarrollo sostenible y consumo, FAJARDO DEL CASTILLO, T., "Los bienes públicos del medio ambiente: el reto de la gestión sostenible de los recursos naturales en la Unión Europea", AFDUAM, núm. 16, 2012, p. 234. Por su parte, FERNANDEZ DE GATTA, D., "Unión Europea: el ambicioso Pacto Verde Europeo y el futuro de la política ambiental de la Unión". En GARCÍA ÁLVAREZ, Gerardo; JORDANO FRAGA, Jesús; LOZANO CUTANDA, Blanca; NOGUEIRA LÓPEZ, A., (Coord..), Observatorio de Políticas Ambientales 2020, Madrid: CIEMAT, 2020, p. 47 , denuncia la dependencia de la industria europea al flujo de extracción de materiales, su transformación en productos y finalmente su eliminación como residuos o emisiones, pues solo el $12 \%$ de los materiales utilizados por la industria procede del reciclado.

9 OSSEWAARDE, M. y OSSEWAARDE-LOWTOO, R., "The EU's Green Deal: A Third Alternative to Green Growth and Degrowth?", Sustainability, núm. 12, 2020, p. 9825. https://doi.org/10.3390/su12239825

${ }^{10}$ BOWEN, A. y FRANKHAUSER, S., "The green growth narrative: Paradigm shift or just spin?", Global Environ. Chang., núm. 21, 2011, pp. 1157-1159.

11 "Communication From the Commission to the European Parliament, The European Council, The Council, The European Economic and Social Committee and the Committee of the Regions", The European Green Deal. COM/2019/640 final.

"Informe

Planeta

Vivo

$2020 "$

WWF,

2020.

Disponible

en 
amenazante reducción de los recursos terrestres y la pérdida de diversidad en el continente ${ }^{13}$. Estas circunstancias han movido a la Unión Europea a girar la vista hacia el "inmenso azul", donde ha anticipado nuevas oportunidades de negocio, nuevas formas para satisfacer las necesidades humanas de alimento y energía y nuevas opciones para el avance de la energía ${ }^{14}$, con vistas a promover el crecimiento de los sectores económicos que tienen relación directa con el océano, lo que ha convenido en llamar "crecimiento azul".

Pero a su vez, los océanos y mares constituyen eje esencial de la vida en la tierra, no solo son esenciales para el sustento del ser humano, también producen el $50 \%$ del oxígeno que respiramos, absorben el $30 \%$ de la emisión global de $\mathrm{CO} 2$, ayudan a combatir el calentamiento global en un $93 \%$ y a mitigar los impactos del cambio climático, contiene una inmensidad de recursos genéticos y biológicos potencialmente útiles para la fabricación de medicinas y alimentos y acoge a los arrecifes de coral y a los manglares, claves para la protección a las costas y para la conservación de ciertos tipos de especies y vegetales ${ }^{15}$.

En este contexto en el que el medio marino constituye promesa de prosperidad económica y a la vez condición para la existencia en la tierra, la realidad es que nuestra forma de producir y de consumir y la manera en que nos hemos estado beneficiando de los recursos marinos ha terminado socavando su propia resiliencia, llevando a la naturaleza a su límite y poniendo en riesgo los mismos servicios y recursos que constituyen un eje básico de nuestro sustento. Existen evidencias de graves y en ocasiones irreparables pérdidas en el capital natural oceánico como consecuencia de un desarrollo económico exacerbado y no sostenible, que está erosionando las reservas de las que este mismo crecimiento depende ${ }^{16}$. Este estrés oceánico ha cristalizado en un efecto degenerativo en cascada que ha desembocado en el rápido declive de la biodiversidad marina, la degradación de hábitats, el incremento de la acidificación de los mares y océanos, la acumulación de basura y plásticos y el aumento de la temperatura del mar ${ }^{17}$.

Ante estas evidencias, en cumplimiento de las prerrogativas autoimpuestas en pos de la sostenibilidad y de los compromisos internacionales adoptados para su cumplimiento ${ }^{18}$, la

https://www.wwf.es/informate/biblioteca_wwf/?55320/Informe-Planeta-Vivo-2020. También en "Blue Growth. Scenarios and drivers for Sustainable Growth from the Oceans, Seas and Coasts. Final Report". Disponible en ECORYS 2012. https://ec.europa.eu/maritimeaffairs/publications/blue-growth-scenarios-and-drivers-sustainablegrowth-oceans-seas-and-coasts_en

13 "The potential of the blue economy", World Bank Group, 2017. Para el Banco Mundial el concepto "economía azul" incorpora tanto la promoción de un crecimiento económico como la inclusión social y la preservación y mejora del medio ambiente, garantizando al propio tiempo la sostenibilidad. Disponible en https://openknowledge.worldbank.org/handle/10986/26843

${ }^{14}$ Comunicación de la Comisión. $\operatorname{COM}(2014) 254$ final/2 "Innovación en la economía azul: aprovechar el potencial de crecimiento y de creación de empleo que encierran nuestros mares y océanos".

15 "Special Report on the Ocean and Cryosphere in a Changing Climate" IPCC, 2019. Disponible en https://www.ipcc.ch/sroce/

16 "Informe Planeta Vivo 2020", WWF, 2020. Disponible en https://www.wwf.es/informate/biblioteca_wwf/?55320/Informe-Planeta-Vivo-2020. En los últimos 50 años nuestra huella ecológica que mide el impacto de nuestro estilo de vida en el planeta ha aumentado por el uso de los recursos en un $190 \%$ : el consumo desenfrenado y el sistema actual de producción son los principales motores detrás de los cambios planetarios sin precedentes que se están viviendo y que tienen una implicación directa en la pérdida de biodiversidad y en los beneficios que la naturaleza nos provee.

17 "Océanos saludables y sostenibles: oportunidades para el sector empresarial en la economía azul. White paper $\mathrm{n}^{\circ}$ 2”, Pacto Mundial. Disponible en https://www.pactomundial.org/sdm_downloads/white-paper-no-2-oceanossaludables-y-sostenibles-oportunidades-para-el-sector-empresarial-en-la-economia-azul/

${ }^{18}$ En este ámbito, FERNANDEZ EGEA, R. M., "Compromisos internacionales en materia del medio ambiente. El Pacto Mundial para el Medio Ambiente: una idea ambiciosa que no pudo ser", en GARCÍA ÁLVAREZ, G., JORDANO FRAGA, J., LOZANO CUTANDA, B. y NOGUEIRA LÓPEZ, A. (Coord.), Observatorio de Políticas Ambientales 2020, Madrid: CIEMAT, 2020, pp. 31 y 32. 
Unión Europea aprobó, en el marco del VI programa de Acción Comunitaria en materia de Medio Ambiente y estrategia temática para el medio marino ${ }^{19}$, la Directiva Marco sobre Estrategia Marina $(\mathrm{DMEM})^{20}$, que recogía como fin primordial la consecución de "un buen estado medioambiental del medio marino" a más tardar en el año 2020 y para cuyo cumplimiento se puso en marcha la Política Marítima Integrada ${ }^{21}$, orientada hacia la economía azul desde la consideración de la interdependencia de todos los sectores de la economía marina $^{22}$ y con miras a su máximo aprovechamiento, con la limitación de la sostenibilidad que imperaba como criterio condicionante.

Así, en el entorno de la Unión y con la vista siempre puesta en la meta del progreso económico tangible, se han sugerido inicialmente "cinco cadenas de valor" potenciales para el crecimiento y el empleo en la economía azul: la energía azul en todos sus ámbitos ${ }^{23}$, la acuicultura, el turismo marítimo, costero y de cruceros, los recursos minerales marinos y la biotecnología azul ${ }^{24}$ y para abordar este potencial y optimizar resultados económicos sin que se vea perjudicado el objetivo de calidad de los océanos y el condicionante de la sostenibilidad, la Unión ha diseñado una estrategia que aborda diversas políticas transversales en las que la búsqueda y el intercambio de información constituyen eje pivotante y pieza clave.

Por un lado, partiendo de la consideración de que el $60 \%$ de los océanos se sitúa fuera de los límites de las jurisdicciones nacionales y que el medio marino constituye un patrimonio común, la Unión se ha propuesto mejorar la gobernanza de los océanos, progresando en el marco de la gobernanza internacional para mejorar la salud de este entorno, reducir las presiones que se ejercen sobre ellos, promover la utilización de sus recursos de manera

\footnotetext{
19 VI programa de Acción Comunitaria en materia de Medio Ambiente "Medio ambiente 2010: el futuro está en nuestras manos" COM (2001) 31 y estrategia temática para el medio marino: Comunicación de la Comisión de 2002 "Hacia una estrategia de protección y conservación del medio ambiente marino" COM (2002) 539 y Comunicación de la Comisión de 2005 "Estrategia temática sobre la protección y la conservación del medio ambiente marino" $\underline{\text { COM }}$ (2005) 504 .
}

${ }^{20}$ La DIRECTIVA 2008/56/CE DEL PARLAMENTO EUROPEO Y DEL CONSEJO de 17 de junio de 2008 por la que se establece un marco de acción comunitaria para la política del medio marino (Directiva marco sobre la estrategia marina). Se aplica a las aguas, el lecho marino y el subsuelo situados más allá de la línea de base que sirve para medir la anchura de las aguas territoriales y que se extienden hasta el límite exterior de la zona en que cada Estado miembro ejerce derechos jurisdiccionales. Además, se aplica a las aguas costeras, su lecho marino y su subsuelo en la medida en la que la Directiva 2000/60/CE no haya abordado los aspectos del estado medioambiental del medio marino. Posteriormente la Comisión ha aprobado la Decisión de la Comisión 2010/477/UE, sobre los criterios y las normas aplicables al buen estado ambiental de las aguas marinas. Dicha Decisión contiene los criterios e indicadores asociados que permiten evaluar el buen estado ambiental, en relación a los 11 descriptores recogidos en el Anexo I de la Directiva.

${ }^{21}$ Véase la Comunicación "Hoja de ruta hacia una Europa eficiente en el uso de los recursos" [COM(2011)571].

${ }^{22}$ La Comisión analiza las cadenas de valor de la economía azul por sectores entre los que incorpora el turismo costero, la extracción de petróleo y gas del mar, la navegación de larga distancia, la navegación de corta distancia, la navegación de recreo y puertos, los servicios de ferri para pasajeros, el turismo de crucero, la pesca, el transporte en aguas interiores, la protección de costas, la energía eólica marina, el seguimiento y vigilancia, la biotecnología azul, la desalinización, la extracción de agregados, productos acuáticos marinos, la extracción de minerales marinos y energías renovables oceánicas.

${ }^{23}$ Se plantea la importancia de la investigación en las diversas tecnologías de producción de energía renovable en la medida que cada combinación de condiciones geográficas y oceanográficas exigen tecnologías diferentes. Así se apuntan junto a la energía eólica, la de las presas mareomotrices, los dispositivos de energía undimotriz y las turbinas subacuáticas así como las planas de conversión de energía térmica oceánica. Véase al respecto Comunicación titulada "Energías renovables: principales protagonistas en el mercado europeo de la energía" COM(2012) 271. En la que se subraya la importancia de la investigación en la energía oceánica.

${ }^{24}$ Se espera que el sector emerja como nicho de mercado centrado en productos de alto valor que se destinen a la sanidad (en este campo se han alcanzado grandes objetivos como ciertos medicamentos antivirales obtenidos de nucleótidos aislados de las esponjas del Caribe o el primer fármaco marino contra el cáncer), a la cosmética y a los biocombustibles (son prometedores los estudios de la utilización de algas para su producción). 
sostenible y fortalecer la investigación y la obtención de datos sobre los océanos a escala internacional ${ }^{25}$. A su vez y con este mismo propósito, en un marco de gobernanza internacional territorialmente más focalizado, se ha favorecido la creación de las estrategias regionales de cuenca en las zonas marítimas colindantes con territorio de la Unión: Mar Adriático y Jónico, Océano Atlántico, El Báltico, el Mar Negro, el Mediterráneo, el Mar del Norte, el Ártico y regiones ultra periféricas, donde se ha promocionado, partiendo de la singularidad de cada región marítima, que los Estados de cada región diseñen y lleven a cabo la estrategia más adecuada para fomentar el crecimiento y desarrollo de su zona que aborde a la misma vez sus propias dificultades, problemáticas y retos específicos, tales como el cambio climático, la seguridad marítima o la degradación de los ecosistemas, entre otros.

Por su parte, a través de la Ordenación del Espacio Marítimo (OEM) la Unión invita a gestionar las aguas con criterios de coherencia y con vistas a que las actividades humanas en el mar se realicen de una forma eficiente, segura y sostenible ${ }^{26}$. Se busca reducir los conflictos entre sectores y crear sinergias entre las actividades que convergen en una misma zona, permitiendo la optimización de su aprovechamiento mediante la cooperación entre países limítrofes que comparten su interés sobre la misma pero con fines diversos (vgr aprovechamiento energético, pesca, conductos submarinos, o rutas de transporte) a la vez que se favorecen mejoras en la protección del medio ambiente en la medida que resulta más fácil identificar con prontitud las amenazas e impactos en la zona.

La Vigilancia Marítima Integrada se orienta a proporcionar métodos de intercambio de datos e información a todas las autoridades interesadas, aunando sectores de dispar naturaleza como control de fronteras, seguridad, control de pesca, aduanas o medio ambiente. Se trata de favorecer la eficacia y mejorar los costes de la vigilancia marítima en un Entorno Común de Intercambio de Información ${ }^{27}$ (ECII) que será puesta a disposición de quienes la necesiten.

La Unión también ha desplegado su Estrategia de Seguridad Marítima (ESMUE) con miras a mantener la prosperidad, la paz y el estado de Derecho en las zonas externas a la jurisdicción nacional y proteger los intereses marítimos estratégicos de la $\mathrm{UE}^{28}$ frente a todo tipo de amenazas que pongan en peligro sus intereses.

En todo este contexto se ha puesto el acento en la importancia del conocimiento del medio marino y de la investigación para poder proteger el océano y favorecer el crecimiento inteligente y sostenible ${ }^{29}$, diseñando una intervención a escala de la Unión en la que se han llevado a cabo acciones como el cartografiado de los fondos marinos, la creación de una

\footnotetext{
${ }^{25}$ Comunicación conjunta al Parlamento Europeo, al Consejo, al Comité Económico y Social Europeo y al Comité de las Regiones: "Gobernanza internacional de os océanos: una agenda para el futuro de nuestros océanos" (SWD (2016) 352 final). Para corregir esta situación reclamando un enfoque intersectorial, internacional y basado en la regulación.

${ }^{26}$ De esta forma la OEM se lleva a cabo de forma transfronteriza e intersectorial y ha dado lugar a la Directiva Marco 2014/89/EU para la Ordenación del Espacio Marítimo.

27 Disponible en https://ec.europa.eu/maritimeaffairs/sites/maritimeaffairs/files/docs/body/cise-ia-study-executivesummary_es.pdf. Véase también Comunicación de la Comisión "Mejor conocimiento de la situación a través de una mayor cooperación entre las autoridades de vigilancia marítima: próximos pasos dentro del entorno común de intercambio de información sobre cuestiones marítimas de la UE". COM (2014) 451 final, Bruselas, 8.7.2014. "Hoja de ruta sobre la vigilancia marítima". Disponible en https://eur-lex.europa.eu/legalcontent/ES/TXT/?uri=COM:2014:0451:FIN

${ }^{28}$ Diseñado por el Consejo Europeo en el año 2014, el Plan de acción sobre la estrategia de seguridad marítima, ha sido revisado en junio de 2018 para reunir los aspectos internos y externos de la seguridad marítima de la Unión.

${ }^{29}$ Red Europea de información del mar EMODNET. Disponible en https://emodnet.eu/en
} 
plataforma de información sobre la investigación marina ${ }^{30}$ o el diseño de perfiles de competencias para la educación y formación de mano de obra cualificada en el sector de la economía azul.

Por último, también en este marco pero desde perspectiva un tanto diferente, en el seno de la Política Marítima Integrada la Unión se ha propuesto optimizar el rol que juegan las comunidades financieras y de inversión en el desarrollo económico, reivindicando la asunción de sus responsabilidades para que en el futuro las decisiones financieras se adopten de conformidad con estándares financieros que guíen a economía hacia la sostenibilidad y promuevan la toma de decisiones responsables en este sector. Se pretende en definitiva orientar el flujo de capital hacia inversiones sostenibles y desplegar estas políticas de sostenibilidad a escala paneuropea. Con esta inquietud se auspició la Declaración principios financieros para la economía azul sostenible ${ }^{31}$, que podrán ser incorporados de forma voluntaria por las entidades de inversión y financiación como criterios de cara a la toma de decisiones respecto de la inversión en uno u otro proyecto ${ }^{32}$, de forma que se promuevan enfoques de financiación medioambiental y socialmente sostenibles mediante la introducción del uso de herramientas como las evaluaciones de riesgo social y medioambiental como indicadores para las principales operaciones financieras, se busca con esta iniciativa que la financiación sirva como eficaz espuela e incentivo que influya en los estándares corporativos en el ámbito social y medioambiental.

Sin embargo, contra toda esperanza, una vez desplegados todos estos esfuerzos en el seno de la Política Marítima común hacia el diseño, planificación y financiación de estas políticas, la evaluación de los avances alcanzados en lo tocante a la mejora de la calidad ambiental de los mares y océanos no han resultado ser muy halagüeños. La Comisión, en su informe evacuado en junio de 2020 sobre la aplicación de la Directiva marco sobre la estrategia marina ${ }^{33}$, constata que en el primer ciclo de aplicación de la directiva no se ha conseguido frenar la pérdida de biodiversidad en Europa, que los hábitats de los fondos marinos de los mares europeos siguen sometidos a una importante presión y que la situación general de las redes tróficas marinas muestra tendencias al deterioro. En este contexto, la Comisión analiza las circunstancias que puedan haber llevado a estos resultados, para terminar señalando entre las razones que están contribuyendo a este decepcionante desenlace el escaso nivel de ambición y voluntad de los Estados a la hora de cumplir y hacer cumplir las medidas planteadas y la falta de participación de los sectores económicos privados.

Sobre la base de estas constataciones, la Comisión realiza sus propuestas de mejora que, lejos de apartarse de la línea ya trazada, abogan por una fórmula continuista que ahora se concentrará por un lado en verificar el cumplimiento efectivo y real de las medidas impuestas y

\footnotetext{
${ }^{30}$ Comunicación de la Comisión $\operatorname{COM}(2014) 254$ final/2 "Innovación en la economía azul: aprovechar el potencial de crecimiento y de creación de empleo que encierran nuestros mares y océanos" Bruselas, 13.5.2014. Sobre competencias y estudios en economía azul, véase https://ec.europa.eu/maritimeaffairs/policy/skills-careerdevelopment_es

31 "Declaration of the sustainable blue economy finance principles". Disponible en https://ec.europa.eu/maritimeaffairs/sites/maritimeaffairs/files/declaration-sustainable-blue-economy-financeprinciples_en.pdf. La declaración recoge 14 principios orientados a complementar el marco normativo existente sobre financiación sostenible.

32 "Informe del Comité de Expertos sobre Financiación Sostenible". Disponible en https://ec.europa.eu/info/publications/sustainable-finance-technical-expert-group_en. Este comité constituye una iniciativa multisectorial centrada en la determinación y propuesta de medidas para el sector financiero con objetivos globales de sostenibilidad.

${ }^{33} \mathrm{COM}(2020) 259$ final INFORME DE LA COMISIÓN AL PARLAMENTO EUROPEO Y AL CONSEJO relativo a la aplicación de la Directiva marco sobre la estrategia marina (Directiva 2008/56/CE).
} 
por otro en la racionalización y simplificación de la aplicación de la directiva y la armonización y disponibilidad de los datos obtenidos. Se propone en definitiva la delimitación de los compromisos y la redefinición de conceptos para favorecer su cumplimiento y ejecución ${ }^{34}$, mientras se apunta hacia la racionalización y coordinación con otras políticas sectoriales, buscando construir puentes sólidos entre la directiva marco y las políticas que regulan las actividades marítimas como la política pesquera común, las iniciativas relacionadas con la energía, la política de transporte marítimo o cualquier otra actividad en los mares y océanos. Sin embargo, contra todo pronóstico el informe de la Comisión no apunta medidas transformadoras, ni se pronuncia respecto de posibles sinergias con otras políticas no relacionadas con las actividades en el mar, como la política agraria o la de residuos plásticos, cuya importante influencia sobre el estado de salud de las aguas europeas ha sido constatada; tampoco avanza medidas correctivas para atajar la contaminación, ni apunta a cambios de rumbo que ofrezcan una respuesta determinante a la necesidad imperiosa de poner freno a esta degradación progresiva y en cascada, que urge una reacción a la altura de las circunstancias.

\section{BALANCE DE LA POLÍTICA MARÍTIMA INTEGRADA 2002-2020. EL IMPERATIVO DE UN CAMBIO DE PARADIGMA REAL}

La estrategia de crecimiento azul de la Unión Europea se presenta como la "gallina de los huevos de oro" que ofrece unas perspectivas de prosperidad y crecimiento prometedoras, planteándose como una opción socialmente interesante que se alinea con varias de las ambiciones de desarrollo sostenible, como el fin de la pobreza (ODS 1), Hambre 0 (ODS 2), trabajo decente y crecimiento económico (ODS 8) o industria e innovación (ODS 9). Sin embargo, estas promesas resultan vacías si no contemplan las nefastas consecuencias que el crecimiento temerario ocasiona sobre nuestro entorno, eje vertebrador de nuestra existencia.

El movimiento en pro de la conservación, restauración y protección de los mares y océanos que la Unión Europea optó por liderar, diseñó una política marítima integrada con la cándida pretensión de dar solución a la crisis ecológica de nuestras aguas marinas mediante la incorporación del enfoque ecosistémico y los criterios de sostenibilidad en la gestión de las actividades humanas sobre los mares europeos, pero manteniendo, e incluso potenciando, el ritmo de crecimiento y desarrollo edificado sobre la economía extractiva seguido hasta la fecha y en definitiva mostrándose poco ambiciosa a la hora de pergeñar iniciativas innovadoras, operar cambios y determinar los correlativos compromisos que aborden de manera radical la fuente de la degradación de los mares y océanos. Las propias instituciones de la Unión han constatado, llegado el término para la comprobación de resultados, que esta política no ha resultado ser suficientemente eficaz para alcanzar los objetivos de calidad medioambiental que se había propuesto.

A la hora de analizar las causas que han motivado tan decepcionantes resultados la Comisión, pese a mostrar cierta complacencia hacia los avances obtenidos, reconoce la

\footnotetext{
${ }^{34} \mathrm{El}$ informe señala la importancia de efectuar una reestructuración de conceptos básicos y de la forma de trabajar, aprovechando al máximo los recursos existentes. Así propone una mejor y más certera delimitación y definición de ciertos conceptos, como el de "calidad medioambiental" como de las obligaciones que se derivan de esta directiva para los Estados. Igualmente insta a la mejora de la recogida de datos y a su complementación con enfoques de modelización, estableciendo métodos estandarizados para monitorear el estado de las aguas y fórmulas de evaluación de los datos homogéneas de cara a la toma de decisiones basada en la evidencia, ya sea coordinando y optimizando el uso de la infraestructura ya existente, ya sea instaurando sistemas eficaces para la recolección de datos interesantes para la realización de estudios científicos, lo que implica además un esfuerzo de homogeneización y uniformidad en cuanto a las fórmulas para publicar esos conocimientos que facilite su máxima divulgación y favorezca en necesario acceso al conocimiento de los mares y de las presiones e impactos de la actividad humana sobre su calidad.
} 
insuficiencia de las medidas adoptadas para atajar eficazmente el problema, pero sin embargo, opta por proponer mejoras en la letra y ejecución de la directiva sin variar el rumbo de la línea trazada por esta. A su vez, si bien la Comisión reconoce la interconexión de la directiva marco con otras reglamentaciones y aboga expresamente por construir "puentes más sólidos documentados por la ciencia entre la Directiva sobre estrategia marina y las políticas que regulan actividades marítimas, (...) cuya expansión no pueden darse a costa e erosionar la resiliencia de los ecosistemas marinos", lejos de las aspiraciones que se anuncian en el Pacto Verde de 2019, ni se avanzan ni se contemplan propuestas "transformadoras" que pongan el acento en la que resulta ser reconocidamente y sin discusión la causa esencial y primordial de esta crisis ecológica: un sistema económico, productivo y de consumo, basado en la extracción y correlativa esquilmación de recursos.

Partiendo de la premisa de que la acción del hombre es la causa directa de la degradación de los ecosistemas marinos, el diseño y planificación de las políticas en pro de la mejora de la calidad de los mares y océanos no puede ignorar el sistema productivo en su conjunto. Reducir el enfoque a las actividades propias de la economía azul, sin prestar la debida atención al resto de sectores y actividades que, no estando relacionadas directamente con los mares y océanos, coadyuvan de forma decisiva en su degradación, no supone una propuesta suficiente para atajar con la determinación que requiere la progresiva degradación descontrolada que está padeciendo nuestro sistema marino.

A su vez, si la industria, el tejido empresarial, el sector energético, etc., son causa directa de la degradación medioambiental, el consumo constituye la razón de ser y el sustento y la fuerza impulsora de aquellos. No abordar de forma prioritaria, decidida y determinante esta circunstancia a la hora de reglamentar, supone nuevamente postergar, incluso obstaculizar, la solución certera y de raíz a esta caída en barrena ecológica.

Sin dejar de valorar positivamente los avances alcanzados en el ámbito del diseño y planificación de una infraestructura útil para el intercambio de información, la transparencia, el avance científico y la cooperación, no podemos evitar hacer notar el decepcionante resultado de la política marítima integrada a nivel de resultados reales de relevancia medioambiental. No debemos obviar la urgencia de atajar de forma directa y sin mayor dilación el escarpado declive de la calidad de nuestros mares, lo que convierte en un imperativo la inmediata mutación del sistema que vaya más allá que una mera revisión de la estrategia; resulta necesario trascender hasta su tuétano y transformar el eje sobre el que gira. Urge un cambio radical de taxonomía ${ }^{35}$ que destaque con preeminencia el ODS 12 y un nuevo enfoque que aborde cambios socioeconómicos en el marco de la transición hacia la sostenibilidad y en el que el principio "pensar primero (y por encima de todo) en la sostenibilidad", impregnando de forma decisiva la toma de decisiones corporativas y devolviendo a los ciudadanos su hegemonía en el proceso de toma de decisiones del mercado ${ }^{36}$. Esto requiere trabajar prioritaria y simultáneamente en dos frentes: el empoderamiento de los consumidores a través de la transparencia, divulgación, accesibilidad de información relativa a la trazabilidad de todos los bienes y servicios objeto de consumo y la liberación a los consumidores del peso que supone el consumo responsable, fomentando los productos y servicios sostenibles con financiación y ayudas que soslayen los mayores gastos que implica este tipo de producción.

\footnotetext{
35 "Informe SOER 2020. Medio Ambiente en Europa, Estado y perspectivas 2020". Resumen ejecutivo. Agencia Europea del Medio Ambiente 2019. Disponible en https://www.eea.europa.eu/publications/soer-2020/\#additionalfiles. Hace hincapié en que no se trata de actuar más, sino de actuar de un modo diferente.
}

36 Almond, R. E. A., GRoOten M. y PETERSEN, T., Living Planet Report 2020 - Bending the curve of biodiversity loss, WWF, Gland, Switzerland, 2020. 
La declaración de principios financieros y la filosofía pragmática que subyace detrás de ella constituyen una manifestación de este espíritu, sin embargo su carácter voluntario vuelve a dejar en el limbo de las "buenas voluntades" unas medidas que ya no tienen espera. Impera una intervención valiente, ambiciosa y certera por parte de la Unión que afronte eficazmente la necesaria transformación de los sistemas sociales-clave responsables de las presiones sobre medio ambiente y nuestros océanos. El Pacto Verde reconoce el protagonismo de los consumidores en la transición ecológica, hace falta la conversión de estos buenos propósitos en políticas reales que incorporen compromisos eficaces para la consecución efectiva de los fines de protección medioambiental.

La realidad nos está demostrando que protección de los océanos y crecimiento azul constituyen ambiciones que pueden resultar absolutamente opuestas. Conviene actuar antes de que nos suceda, cual fábula de Samaniego, como a la lechera, se rompa nuestro cántaro y con él nuestras posibilidades de subsistencia. Es preciso forzar sinergias doblegando las pretensiones económicas cortoplacistas para que respondan prioritariamente a criterios de sostenibilidad, en este ámbito los derechos de información y participación de los ciudadanos constituyen herramientas estratégicas clave para la ecologización de las decisiones del mercado.

\section{REFERENCIAS BIBLIOGRÁFICAS}

- BOWEN, A. y FRANKHAUSER, S., "The green growth narrative: Paradigm shift or just spin?”, Global Environ. Chang., núm. 21., 2011, pp. 1.157-1.159.

- DÍEZ DE VELASCO VALLEJO, M., "Aspectos jurídicos actuales de la protección del medioambiente en la Comunidad Europea y, en especial, la contribución de su Tribunal de Justicia”, en RODRÍGUEZ IGLESIAS, G. y LIÑÁN NOGUERAS, D., (Coord.), El derecho comunitario europeo y su aplicación judicial, Civitas, Madrid, 1993. pp. 10031028.

- ESPÓSITO MASSICCI, C. y FERNÁNDEZ EGEA, R. M., "La protección del medio ambiente a través de los derechos exclusivos y el derecho de la competencia. A propósito de la sentencia del Tribunal de Justicia de las Comunidades Europeas de 23 de mayo de 2000", Gaceta jurídica de la Unión Europea y la competencia, núm. 211, 2001.

- FAJARDO DEL CASTILlO, T., "Los bienes públicos del medio ambiente: el reto de la gestión sostenible de los recursos naturales en la Unión Europea”, AFDUAM, núm. 16, 2012, p. 234.

- FERNANDEZ EGEA, R.M. "Compromisos internacionales en materia del medio ambiente. El Pacto Mundial para el Medio Ambiente: una idea ambiciosa que no pudo ser", en GARCÍA ÁlVAREZ, G., JORDANO FRAGA, J., LOZANO CUTANDA, B. y NOGUEIRA LÓPEZ, A., (Coord.), Observatorio de Políticas Ambientales 2020, CIEMAT, Madrid, 2020, pp. 21-41.

- FERNÁNDEZ DE GATTA SÁNCHEZ, D., "La política ambiental comunitaria: especial referencia a los programa ambientales", Revista de Instituciones Europeas, núm. 3, 1985, pp. 723-753.

- FERNANDEZ DE GATTA, D., "Unión Europea: el ambicioso Pacto Verde Europeo y el futuro de la política ambiental de la Unión", en GARCÍA ÁLVAREZ, G., JORDANO FRAGA, J., LOZANO CUTANDA, B. y NOGUEIRA LÓPEZ, A., (Coord.), Observatorio de Políticas Ambientales 2020, CIEMAT, Madrid, 2020, pp. 41 -86. 
- GARCÍA DE ENTERRÍA, E., GONZÁLEZ CAMPOS, J. D. y MUÑOZ MACHADO, S. (Dir.), Tratado de Derecho Comunitario Europeo, núm.. III, Civitas, Madrid, 1986.

- LIÑÁN NOGUERAS, D. J. y MARTÍN RODRÍGUEZ, P. J., "Reflexiones sobre los derechos fundamentales de la Unión europea a la luz del Tratado de Lisboa", en BADIA MARTí, A. M., PIGRAU SOlÉ, A. y OLESTI RAYO, A., (Coord.), Derecho Internacional y comunitario ante los retos de nuestro tiempo. Homenaje a la profesora Victoria Abellán Honrubia, Marcial Pons, Madrid, 2009.

- MANGAS MARTÍN, A., "El compromiso con los derechos fundamentales", en MANGAS MARTÍN, A., GONZÁLEZ ALONSO, L. N., LÓPEZ ESCUDERO, M., MARTÍN Y PEREZ DE NANClARES, J. y SOBRINO HEREDIA, J. M., Carta de los Derechos Fundamentales de la Unión Europea. Comentario artículo por artículo, Fundación BBVA, Bilbao, 2008. pp. 809-850.

- MARTÍN MATEO, R., "El ambiente en la CEE", Noticias de la Unión Europea, núm. 14, 1986.

- OSSEWAARDE, M., y OSSEWAARDE-LOWTOO, R., “The EU's Green Deal: A Third Alternative to Green Growth and Degrowth?”, Sustainability, núm. 12, 2020, p. 9.825. Disponible en https://doi.org/10.3390/su12239825

- VITORINO, A., "La Cour de justice et les droits fondamentaux depuis la proclamation de la Charte", en COLNERIC, N., PUISSOCHET, J. P., RUIZ-JARABO Y COLOMER, D. y EDWARDS, D. V., Une communauté de droit: Festschrift für Gil Carlos Rodríguez Iglesias, BWV Berliner Wissenshafts, Berlin, 2003. 